F. Geusa, A. Agnes, F. Maloberti: "Use of Chopper-Notch Modulator in Chopper Amplifiers for Replica Images Cancellation"; 15th IEEE Int. Conf. on Electronics, Circuits and Systems, ICECS 2008, St. Julien's, August 31-September 3, 2008, pp. 566-569.

(C)20xx IEEE. Personal use of this material is permitted. However, permission to reprint/republish this material for advertising or promotional purposes or for creating new collective works for resale or redistribution to servers or lists, or to reuse any copyrighted component of this work in other works must be obtained from the IEEE. 


\title{
Use of Chopper-Notch Modulator in Chopper Amplifiers for Replica Images Cancellation
}

\author{
Francesco Geusa, Andrea Agnes, Franco Maloberti \\ Department of Electronics, University of Pavia \\ via Ferrata, 1, 27100 Pavia, Italy \\ Email: francesco.geusa01@ ateneopv.it, andrea.agnes@unipv.it, franco.maloberti@unipv.it
}

\begin{abstract}
A method for cancelling the replica image limiting the chopper stabilization technique is presented. The modulation of the $1 / f$ noise at the chopping frequency and its multiple are conventionally reduced by a low-pass filter and, possibly by a notch filter. The method presented here combines the second chopper and the notch filter by using a simple SC circuit that enables cancelling the replica images. The method just requires a simple local loop for reducing the amplified offset to a reasonable level and this is obtained with a simple modification of the amplifier used in the chopper scheme. Simulation results show the expected benefits.
\end{abstract}

\section{INTRODUCTION}

Many instrumentation applications require using amplifiers with ultra high-gain and virtually zero offset and $1 / f$ noise for processing the signal that sensors produce at very low level. The use of nested architectures obtains high gain and, at the same time, enables compensation. The offset is faced by the auto-zero or the chopper stabilized approach. Both methods are also effective against the $1 / f$ noise. The auto-zero technique is more performant than the chopper approach but, unfortunately, it requires to use large auto-zero capacitances to obtain a low $k T / C$ contribution. On another hand, the chopper stabilization method generates images of offset and $1 / f$ noise at the chopping frequency and its multiples. These spurs are unwanted and must be filtered out by on-chip low pass or notch filters.

A possible architecture with three stages is the nested scheme shown in Fig. 1. The low frequency gain is $A_{0}=A_{3}+A_{2} \cdot A_{3}+A_{1} \cdot A_{2} \cdot A_{3}$. The feedthrough path through $A_{3}$ sustain the gain at high frequency. The offset and the $1 / f$ noise of the first amplifier, $A_{1}$, can be reduced by the auto-zero or the chopper method [1]. The offset and the l/f noise of $A_{2}$ and $A_{3}$ are referred to the input attenuated by a factor $A_{1}$ and $A_{1} \cdot A_{2}$ respectively, therefore these contributions are negligible.

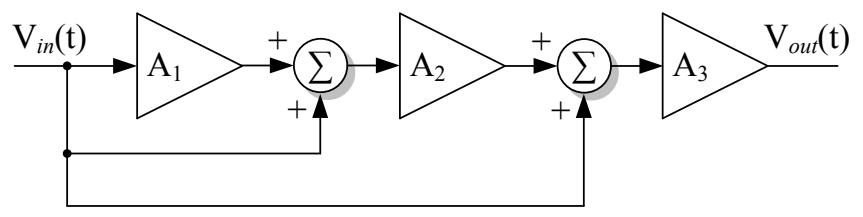

Fig. 1. Conventional scheme of a nested amplifier.

One of the solutions used to cancel the offset is the auto- zero [4] method. It requires a time-slot during which the amplifier is not used. The inputs are shorted and an auxiliary gain stage is suitably driven to zero the output. The auto-zero signal is stored on a capacitor for the successive amplification phase. The method can also use the solution of Fig. 2(b) that stores the offset on $C_{1}$ placed in series with the input. The value of the capacitor and the auto-zero rate determine the noise floor limit that is $\sqrt{k T /\left(C_{1} \cdot f_{a z}\right)}$. For obtaining a noise floor of $50 \mathrm{nV} / \sqrt{\mathrm{Hz}}$ with $f_{a z}=10 \mathrm{kHz}$ it is necessary to use $164 p F$, an area consuming value for onchip implementation.

(a)

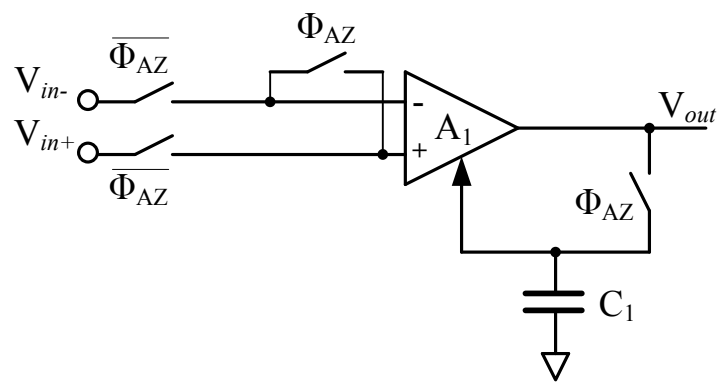

(b)
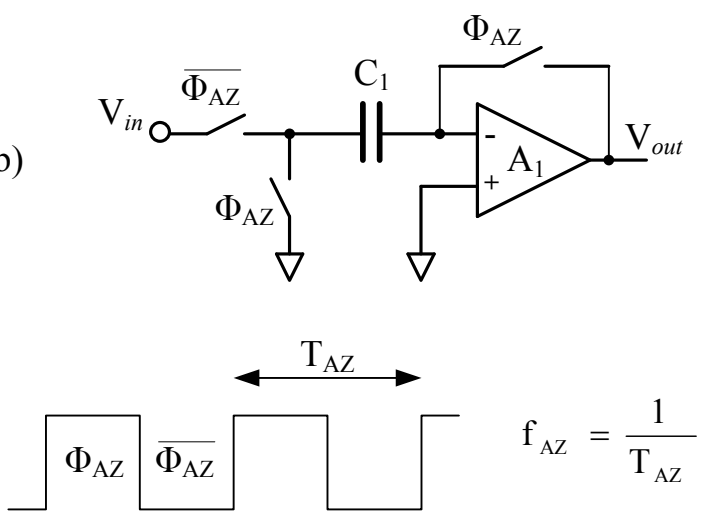

Fig. 2. Schemes for auto-zero.

The above motivates the use of the chopper stabilization method and to study possible solutions that resolve the problem of the spur images. The method described in this paper is an hybrid solution that uses the chopper method before the amplifier and exploits an auto-zero like approach to reconstruct the signal in the base-band. The analysis and simulations verify 
the benefits of the proposed method.

\section{CHOPPER TECHNIQUE AND LIMITS}

A chopper stabilized amplifier multiplies the input signal by a square wave for moving the band limited input spectrum around the chopping frequency and its multiples. The operation separates the signal from offset and the $1 / f$ noise so that the spectra are like the ones shown in Fig. 3. The input spectrum is the one in Fig. 3(b). After chopping and the addition of $v_{n}(t)$ that includes the offset $v_{o s}$ and the $1 / f$ noise $v_{f}(t)$ we have the spectrum of Fig. 3(c). After the second modulator the spectrum becomes the one of Fig. 3(d). It results that spurs at $f_{c h}$ and its multiples deteriorate the result. A lowpass filter can possibly reduce the amplitude of the offset and the $1 / f$ contributions.

(a)

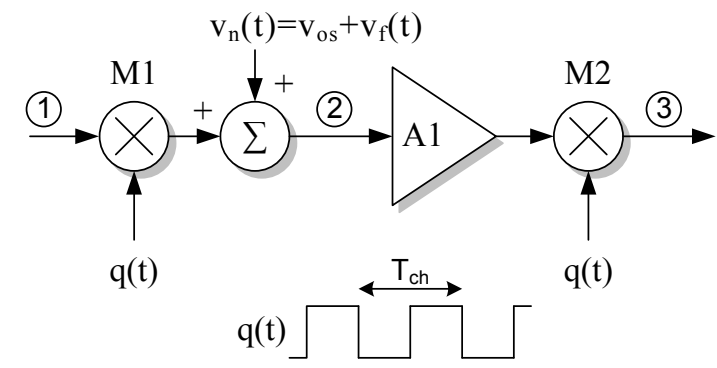

(b)

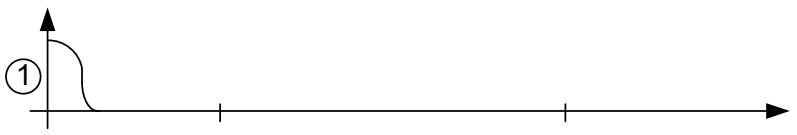

(c)

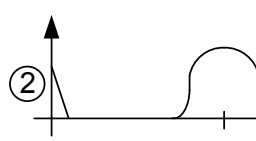

(d)

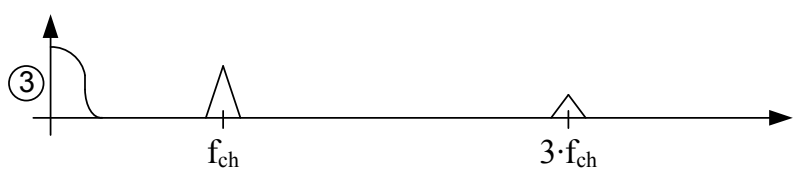

Fig. 3. a) Schemes of chopping solution and spectra b) of the input signal, c) of the signal after first modulation and d) of the output signal.

The method has been simulated by using a test $1 / f$ noise whose spectrum is shown in Fig. 4(a). The spectrum of the output spur, after modulation, is the one in Fig. 4(b) and after a first order low pass filter with cut-off frequency at $f_{c h} / 10$ becomes the one of Fig. 4(b). It is evident that the residual at $f_{c h}$ is still high and, likely, many applications cannot admit the result.

A possible remedy is use a chopping signal with, intentionally, a jitter. The effect is to blur the spur power and to spread it around $f_{c h}$ and its multiples. As shown in [2] the method works but the level of the noise floor increases and, therefore, there is a trade-off between level of the noise floor and the presence of spur tones. The spur tones can also be removed by a notch filter, that replaces or is placed after the low-pass filter [6], centered at $f_{c h}$ and multiples. The possible
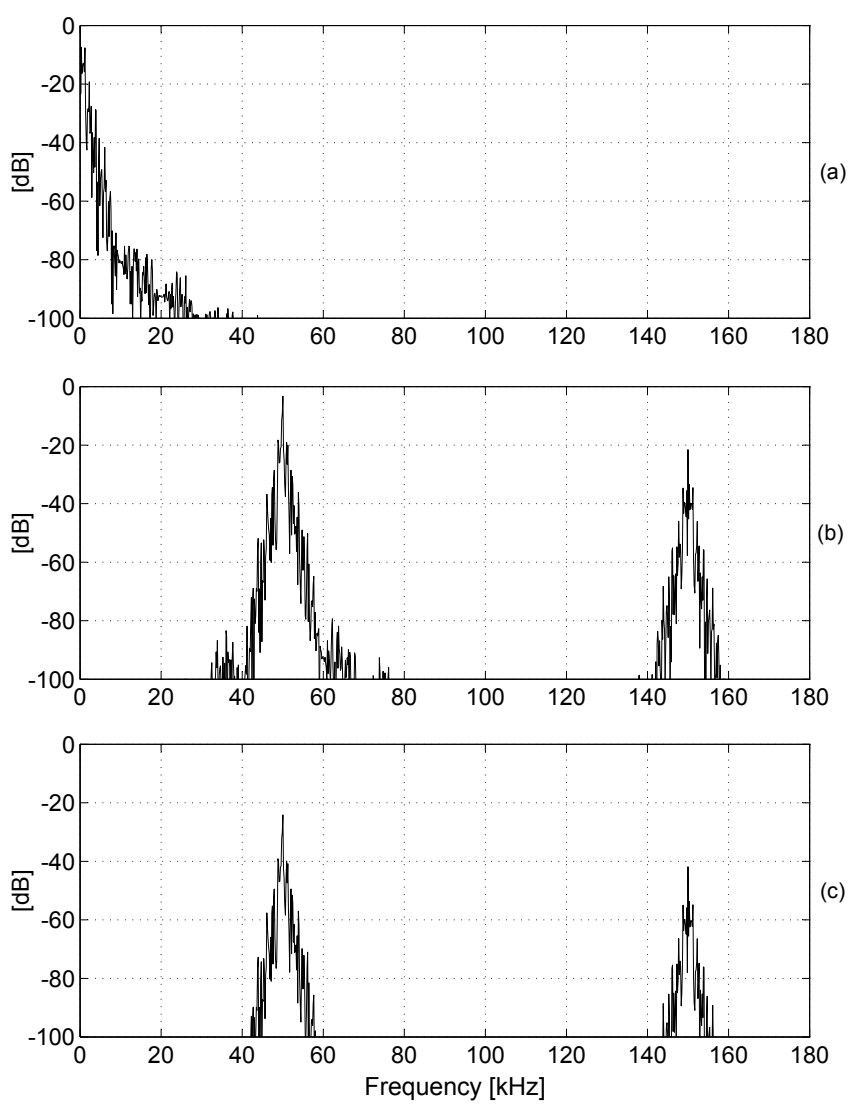

Fig. 4. Spectra of the a) $1 / f$ noise used for simulations b) output of the second modulator M2 and, c) output of the low-pass filter with cut-off frequency at $f_{c h} / 10$.

notch response is shown in Fig. 5 that must be provided by an active or a passive SC scheme.

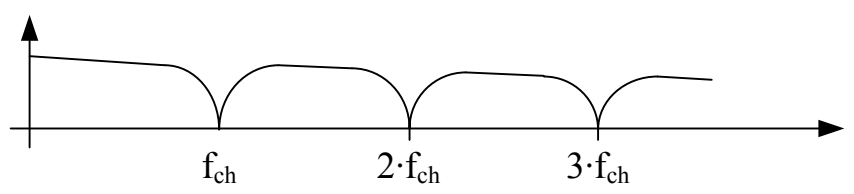

Fig. 5. Possible notch response.

\section{PROPOSED SC CHOPPER-NOTCH}

The proposed solution is based on the following observations/assumptions:

- the chopping frequency is higher than the signal band and aliasing is not a problem;

- the chopped path takes care of the low-frequency part of the input (as it is for the auto-zero case);

- the input is transformed into a pseudo-square wave, while the offset is a dc term.

Therefore, the goal of the circuit is to reject the $d c$ and to reveal the peak-to-peak amplitude of the square wave.

It is well known that a $d c$ component is rejected with a capacitive coupling and that the peak-to-peak amplitude of a 
square wave results from subtracting the negative value from the positive value.

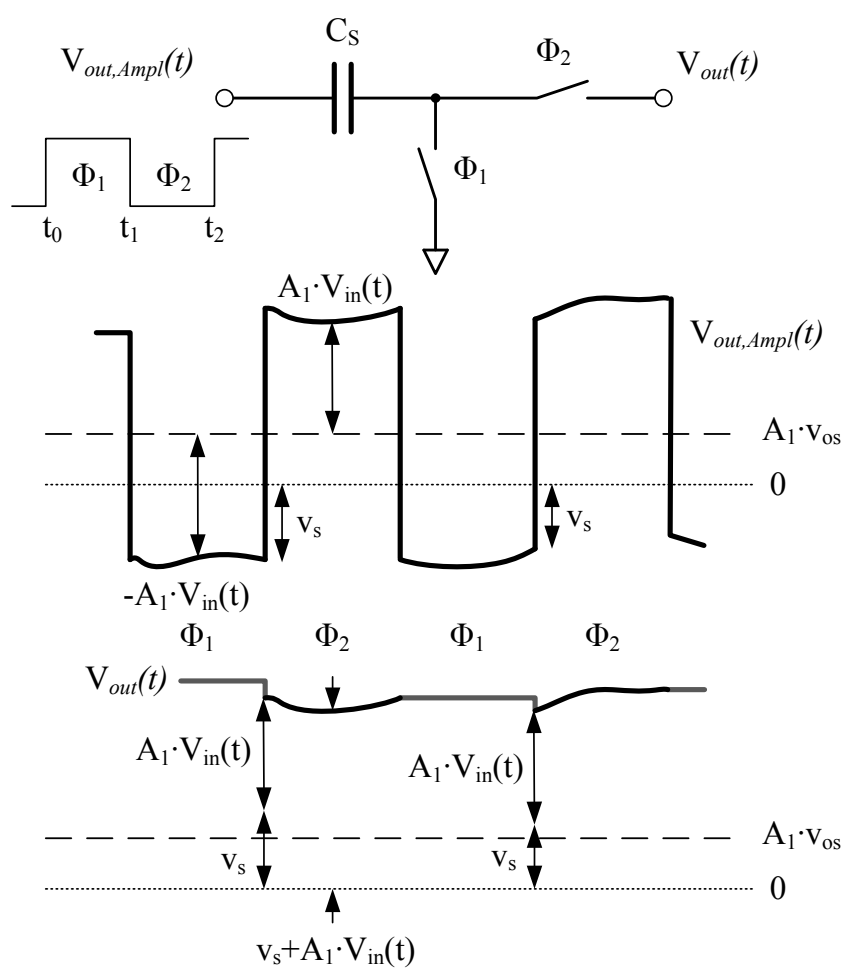

Fig. 6. Chopper-notch with SC structure.

These functions are obtained by the simple circuit of Fig. 6 . During the phase $\Phi_{1}$, the signal equal to $A_{1}\left[-V_{\text {in }}\left(t_{1}\right)+V_{o s}\right]$ is sampled on the capacitor $C_{s}$, while during the phase $\Phi_{2}$ the capacitor acts as a level shift giving rise to the output $V_{\text {out }}$ at the end of $\Phi_{2}\left(t=t_{2}\right)$ equal to

$$
V_{\text {out }}\left(t_{2}\right)=A_{1}\left[V_{\text {in }}\left(t_{1}\right)+V_{\text {in }}\left(t_{2}\right)\right]
$$

Indeed, if in addition to the offset there is also a $1 / f$ term, $V_{f}$ the output voltage becomes

$$
V_{\text {out }}\left(t_{2}\right)=A_{1}\left[V_{\text {in }}\left(t_{1}\right)+V_{\text {in }}\left(t_{2}\right)-V_{f}\left(t_{1}\right)+V_{f}\left(t_{2}\right)\right]
$$

That corresponds to a $\left(-1+z^{-1}\right)$ high-pass filter of $V_{f}(t)$ in the sampled-data domain, as it is obtainded by the auto-zero method.

\section{LOCAL CONTROL OF THE OFFSET}

The $d c$ coupling established by the simple circuit of Fig. 7 naturally removes the offset but, if the gain $A_{1}$ is large, a non negligible offset give rise to an amplified result that can saturate the output of the amplifier. Therefore, it is necessary to properly control the frequency response of the amplifier. Indeed, it is not necessary to remove the $d c$ but it is just enough to limite the gain to a level that avoids the output saturation.

A possible conceptual solution is shown in Fig. 8. It is made by two amplifiers one with the full gain and the other with

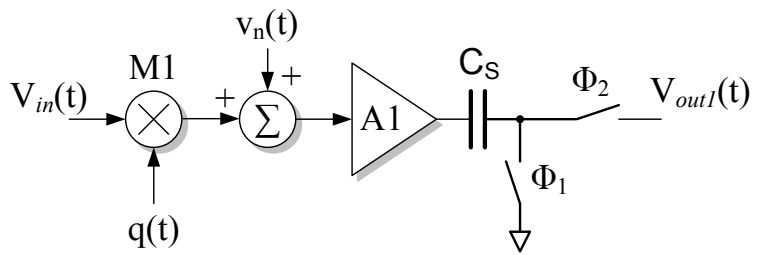

Fig. 7. Circuit for offset cancellation.

reduced gain that add their contributions to obtain the output. The low gain inputs serve for a local loop that limits the low frequency gain.

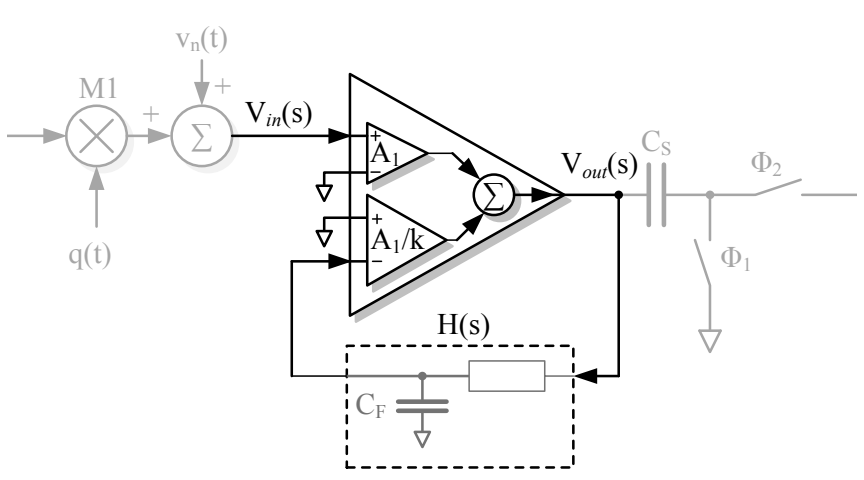

Fig. 8. Amplifier with local loop for offset control.

By inspection of the scheme it results

$$
V_{\text {out }}(s)=V_{\text {in }}(s) \frac{k}{\frac{k}{A_{1}}+H(s)}
$$

if $H(s)$ is a low pass filter, $H(s)=1 /(1+s \tau)$, then

$$
A(s)=\frac{V_{\text {out }}(s)}{V_{\text {in }}(s)} \simeq k \frac{1+s \tau}{1+s \tau \frac{k}{A_{1}}}
$$

denoting a gain by $k$ at $d c$ and a zero-pole pair separated by $k / A_{1}$. If the pole occurs before the dominant pole of the amplifier, then the frequency response is like the diagram of Fig. 9. The offset and the $1 / f$ noise is amplified by $k$ and the chopped signal by $A_{1}$.

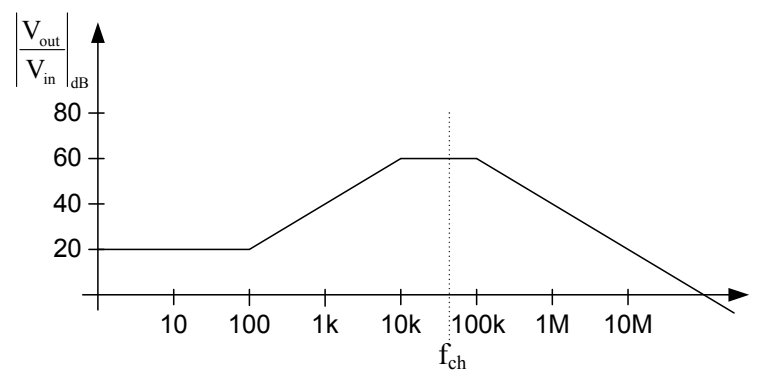

Fig. 9. Typical frequency response of the chopped amplifier.

Assuming a chopper frequency of $50 \mathrm{kHz}$, as shown in Fig. 9, and a signal bandwith of $20 \mathrm{kHz}$, the flat gain bandwith 
has to be at least $40 \mathrm{kHz}$ around of the chopper frequency. Therefore the dominant pole of the amplifier $A_{1}$ can be set at $100 \mathrm{kHz}$ and if its gain is $60 \mathrm{~dB}$ the $f_{t}=100 \mathrm{MHz}$. A reasonable position of the pole of $\mathrm{A}(\mathrm{s})$ is at $10 \mathrm{kHz}$. With the zero at $100 \mathrm{~Hz}$ the low frequency gain of the amplifier is $20 \mathrm{~dB}$ that is a limited value for offset of few $\mathrm{mV}$. The design strategy can lead to different positions of zero and poles; however the above figures give an indication on the design of the block $\mathrm{H}(\mathrm{s})$.

\section{CIRCUITS FOR LOCAL LOOP}

The previous section shows the need of designing a low pass filter with a relatively low cut off frequency (around $100 \mathrm{~Hz}$ ). The problem has been faced in many biomedical applications showing that the solution is not trivial but possible to achieve.

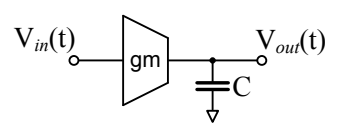

Fig. 10. gm-C low pass filter.

For a cut off frequency of $100 \mathrm{~Hz}$ the equivalent time constant is $1.4 \mathrm{msec}$. The resistance can be realized with high-resistive poly or with a transconductor and a possible value is in the few $\mathrm{M} \Omega$ range. Assuming to obtain $5 \mathrm{M} \Omega$ the required capacitance is $320 \mathrm{pF}$. This value is large for an integrated implementation; however, techniques that multiply grounded capacitors can be used. Fig. 11 shows the transistor implementation of the scheme proposed in [7]. The current on the capacitance $C_{i}$ is measured and amplifier by a current mirror with gain $\alpha$. Therefore the optained capacitance is $C_{i} \cdot(1+\alpha)$. By using $\alpha=49$ the capacitance to be integrated is reduced to $6.4 \mathrm{pF}$, a large but affordable value.

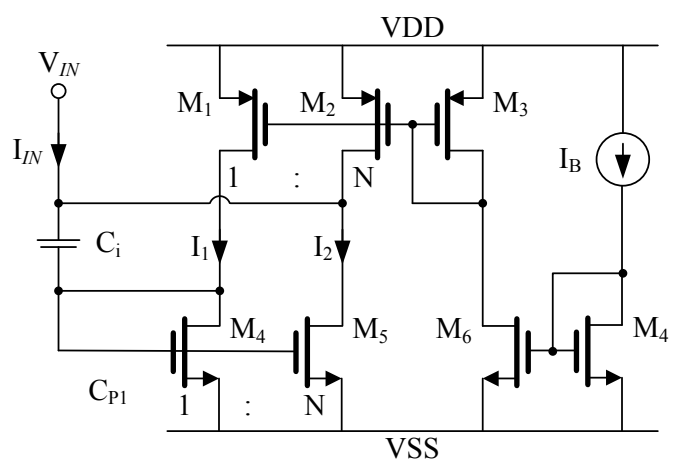

Fig. 11. Circuit for capacitance multiplier.

\section{SyStem Simulation}

Fig. 12 shows the complete scheme of the nested amplifier made by two stages with gain $A_{1}$ and $A_{2}$. Therefore the $d c$ gain becames $\left(A_{2}+A_{1} \cdot A_{2}\right)$. The amplifier $A_{1}$ has in front the first chopper and is followed by the chopper-notch proposed circuit. Moreover a local loop obtain a limited gain of the low frequency input. The operation of the circuit has been simulated at the behavioural and transistor level to demonstrate the proposed technique.

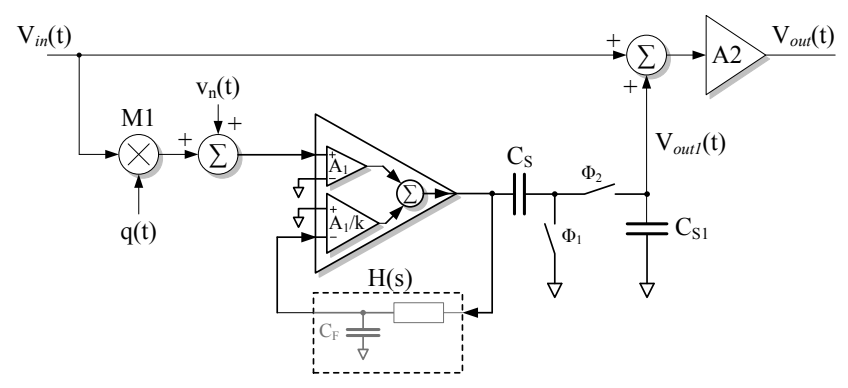

Fig. 12. Complete amplifier.

Fig. 13 shows the spectrum at the output under the same conditions used in Fig. 6. The offset (equal to $4.3 \mathrm{mV}$ ) is completely removed while the $1 / f$ noise is strongly attenuated by more than $60 \mathrm{~dB}$. The chopping action that has a continuous time path during $\Phi_{2}$ causes the replicas at multiples of the $f_{c h}$ that are below $90 \mathrm{~dB}$ in the worst case.

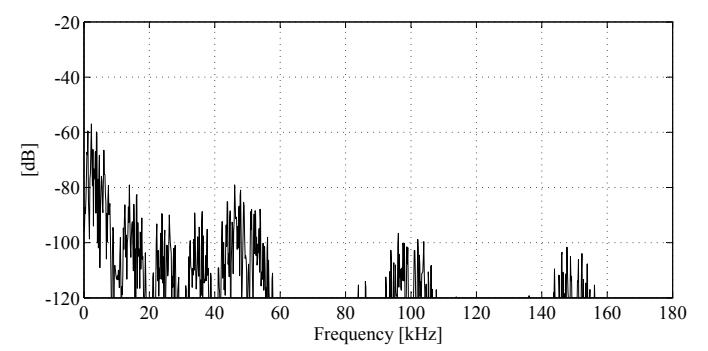

Fig. 13. Spectrum of the output caused by $1 / \mathrm{f}$ noise.

\section{ACKNOWLEDGEMENTS}

This work is supported by FIRB project, Italian National Program \#RBAP06L4S5.

\section{REFERENCES}

[1] A. Thomsen, D. Kasha, W. Lee, "A Five Stage Chopper Stabilized Instrumentation Amplifier Using Feedforward Compensation", IEEE Symposium on VLSI Circuits Digest of Tecnical Papers, pp. 220-223, Jun 1998.

[2] A. Agnes, F. Maloberti, G. Martini, "Improved chopper stabilized amplifier for offset and 1/f noise cancellation", IEEE International Conference on Electronics, Circuits, and Systems (ICECS'06), pp. 529-532, Dec 2006.

[3] L. Toth and Y.P.Tsividis, "Generalization of the principle of chopper stabilization", IEEE Trans. Circuits Syst. I, Fundam. Theory Appl., vol.50, pp. 975-983, Aug 2003.

[4] T. Yoshida, Y. Masui, T. Mashimo, M. Sakaki, and A. Iwata, "A 1 V supply $50 \mathrm{nV} / \sqrt{\mathrm{Hz}}$ noise PSD CMOS amplifier using noise reduction technique of autozeroing and chopper stabilization", Dig. Tech. Papers 2005 Symp. VLSI Circuits, pp. 118-121, 2005.

[5] C.C. Enz, G.C. Temes, "Circuit techniques for reducing the effects of opamp imperfections: Autozeroing, correlated double sampling, and chopper stabilization", Proc. IEEE, vol. 84, pp. 1584-1614, Nov 1996.

[6] R. Burt, J. Zhang, "A Micropower Chopper-Stabilized Operational Amplifier Using a SC Notch Filter With Synchronous Integration Inside the Continuous-Time Signal Path", IEEE Journal of Solid-State Circuits, pp. 2729-2736, Dec 2006.

[7] S. Solís-Bustos, J. Silva-Martínez, F. Maloberti, E. Sánchez-Sinencio, "A 60-dB Dynamic-Range CMOS Sixth-Order 2.4-Hz Low-Pass Filter for Medical Applications", IEEE Transactions on Circuits and systems II, pp. 1391-1398, Dec 2000. 